\title{
Red Flags for Multiple System Atrophy
}

\author{
Martin Köllensperger, MD, ${ }^{1}$ Felix Geser, MD, PhD, ${ }^{2}$ Klaus Seppi, MD ${ }^{2}$
}

Michaela Stampfer-Kountchev, MD, ${ }^{2}$ Martin Sawires, MD ${ }^{2}$ Christoph Scherfler, MD, ${ }^{2}$ Sylvia Boesch, MD, ${ }^{2}$ Joerg Mueller, MD, ${ }^{2}$ Vasiliki Koukouni, MD ${ }^{3}$ Niall Quinn, MD, Maria Teresa Pellecchia, MD, ${ }^{4}$ Paolo Barone, MD, ${ }^{4}$ Nicole Schimke, MD, ${ }^{5}$ Richard Dodel, MD,${ }^{5}$ Wolfgang Oertel, MD, ${ }^{5}$ Erik Dupont, MD, ${ }^{6}$ Karen Østergaard, MD, PhD, ${ }^{6}$ Christine Daniels, MD, Günther Deuschl, MD, ${ }^{7}$ Tanya Gurevich, MD ${ }^{8}$ Nir Giladi, MD ${ }^{8}$ Miguel Coelho, MD, ${ }^{9}$ Cristina Sampaio, MD, ${ }^{9}$ Christer Nilsson, MD,${ }^{10}$ Håkan Widner, MD,${ }^{10}$ Francesca Del Sorbo, MD ${ }^{11}$ Alberto Albanese, MD ${ }^{11}$ Adriana Cardozo, MD, ${ }^{12}$ Eduardo Tolosa, MD,${ }^{12}$ Michael Abele, MD, ${ }^{13}$ Thomas Klockgether, MD,${ }^{13}$ Christoph Kamm, MD,${ }^{14}$ Thomas Gasser, MD, ${ }^{14}$ Ruth Djaldetti, MD, ${ }^{15}$ Carlo Colosimo, MD ${ }^{16}$ Giuseppe Meco, MD, ${ }^{16}$ Anette Schrag, MD, PhD, ${ }^{3}$ Werner Poewe, $\mathrm{MD}^{2}$, and Gregor K. Wenning, MD, PhD, ${ }^{1 *}$ on behalf of the European MSA Study Group (EMSA-SG)

\author{
${ }^{1}$ Section for Clinical Neurobiology, Department of Neurology, Innsbruck Medical University, Austria \\ ${ }^{2}$ Department of Neurology, Innsbruck Medical University, Austria \\ ${ }^{3}$ Institute of Neurology, University College, London \\ ${ }^{4}$ Department of Neurological Sciences, University Federico II, Naples, Italy \\ ${ }^{5}$ Department of Neurology, Philipps-University, Marburg, Germany \\ ${ }^{6}$ Department of Neurology, University Hospital, Aarhus, Denmark \\ ${ }^{7}$ Department of Neurology, Christian-Albrechts-University, Kiel, Germany \\ ${ }^{8}$ Department of Neurology, Sourasky Medical Center, Tel Aviv, Israel \\ ${ }^{9}$ Department of Neuroscience, Faculdade de Medicina de Lisboa, Portugal \\ ${ }^{10}$ Department of Clinical Neuroscience, University of Lund, Sweden \\ ${ }^{11}$ Instituto Carlo Besta, Milano, Italy \\ ${ }^{12}$ Department of Neurology, University of Barcelona, Spain \\ ${ }^{13}$ Department of Neurology, University of Bonn, Germany \\ ${ }^{14}$ Department of Neurodegenerative Diseases, University of Tübingen, Tübingen, Germany \\ ${ }^{15}$ Department of Neurology, Rabin Medical Centre, Petach-Tiqva, Israel \\ ${ }^{16}$ Department of Neuroscience, University La Sapienza, Rome, Italy
}

\begin{abstract}
The clinical diagnosis of multiple system atrophy (MSA) is fraught with difficulty and there are no pathognomonic features to discriminate the parkinsonian variant (MSAP) from Parkinson's disease (PD). Besides the poor response to levodopa, and the additional presence of pyramidal or cerebellar signs (ataxia) or autonomic failure as major diagnostic criteria, certain other clinical features known as "red flags" or warning signs may raise the clinical suspicion of MSA. To study the diagnostic role of these features in MSA-P versus PD patients, a standardized red flag check list (RFCL) developed by the European MSA Study Group (EMSA-SG) was administered to 57 patients with probable MSA-P and 116 patients with probable PD diagnosed according to established criteria. Those red flags with a specifity over $95 \%$ were selected for further analysis.
\end{abstract}

Factor analysis was applied to reduce the number of red flags. The resulting set was then applied to 17 patients with possible MSA-P who on follow-up fulfilled criteria of probable MSA-P. Red flags were grouped into related categories. With two or more of six red flag categories present specificity was $98.3 \%$ and sensitivity was $84.2 \%$ in our cohort. When applying these criteria to patients with possible MSA-P, 76.5\% of them would have been correctly diagnosed as probable MSA-P $15.9( \pm 7.0)$ months earlier than with the Consensus criteria alone. We propose a combination of two out of six red flag categories as additional diagnostic criteria for probable MSA-P. (C) 2008 Movement Disorder Society

Key words: multiple system atrophy; diagnosis; red flags; warning signs
*Correspondence to: Dr. Gregor Wenning, Section for Clinical Neurobiology, Department of Neurology, Innsbruck Medical University, Anichstrasse 35, 6020 Innsbruck, Austria.

E-mail: gregor.wenning@i-med.ac.at
Received 21 May 2007; Revised 3 September 2007; Accepted 22 January 2008

Published online 28 April 2008 in Wiley InterScience (www. interscience.wiley.com). DOI: 10.1002/mds.21992 
Multiple system atrophy (MSA) is a sporadic neurodegenerative disorder, histopathologically characterized by $\alpha$-synuclein positive glial cytoplasmic inclusions (GCIs). Clinical presentation is variable, featuring parkinsonian, cerebellar, and autonomic features in any combination.

According to the Consensus criteria, patients with MSA are clinically classified according to the predominant motor presentation into cerebellar (MSA-C) and parkinsonian (MSA-P) subtype. ${ }^{1}$ However, there are few pathognomonic features to discriminate the parkinsonian variant from Parkinson's disease (PD), resulting in considerable underrecognition of MSA in early disease states. There is certainly a need for improved screening instruments, as an accurate diagnosis of MSA is important for several reasons including prognostic and therapeutical implications.

Additional tools, such as cardiovascular autonomic function tests, anal sphincter electromyography, MIBG scintigraphy of cardiac sympathetic terminals, SPECT scans of dopamine transporters, diffusion weighted magnetic resonance tomography (DWI), have been reported to be useful in this differential diagnosis. However, most studies are limited to advanced disease stages, providing no information on diagnostic value for early diagnosis. In addition, many patients are being referred to movement disorder specialists only after the first atypical signs occur, such as rapid progression or poor response to levodopa.

Besides the poor response to levodopa (L-dopa), and the additional presence of pyramidal or cerebellar signs or autonomic failure as major diagnostic clues, other features (red flags) such as orofacial dystonia or stridor may raise suspicion of $\mathrm{MSA}^{2-5}$

In the present multicenter study a standardized red flag check list (RFCL) developed by the European MSA Study Group (EMSA-SG) was administered to patients with MSA-P and PD diagnosed according to established criteria. The primary objective was to determine the frequencies and the diagnostic role of potential red flags for MSA in these patient cohorts.

\section{PATIENTS AND METHODS}

\section{Patients}

Fifty-seven patients with probable MSA-P and 116 patients with probable PD matched for age, sex, and disease duration were included in the study at 15 EMSA-SG centers. In addition, the RFCL was also administered to 17 patients with a diagnosis of possible MSA-P at baseline who fulfilled the Consensus criteria for probable MSA- $\mathrm{P}^{1}$ at follow-up in order to determine the diagnostic role of red flags in early disease stages. Follow-up visits were performed every 6 months for up to 2 years. Written informed consent was obtained from each patient. RFCL administration was approved by the investigational review boards.

\section{METHODS}

On the basis of a detailed clinical history and examination, diagnosis was made according to established criteria. MSA-P was diagnosed according to the Consensus criteria, ${ }^{1}$ PD according to the United Kingdom Parkinson's Disease Society Brain Bank criteria. ${ }^{6}$ All patients were rated on the Hoehn and Yahr (H\&Y) Parkinson's disease staging system. A comprehensive EMSA-SG red flag check list drawn up based on literature review and expert opinion (Table 1) was completed by the examiners according to standardized instructions. Most of the features were operationally defined (e.g. rapid progression) while some were based on clinical judgement alone (e.g. orofacial dystonia or severe dysphonia).

\section{Statistical Analysis}

Data collected at the participating centers were entered in a central ACCESS database held at the Innsbruck site and analyzed using SPSS 14.0 for Windows (SPSS Inc, USA). The significance level was set at $P \leq 0.05$ and corrected for multiple testing.

Data are reported as mean ( \pm standard deviation) or median (and the interquartile range) depending on data distribution. Student's t-test was used for comparison of age and disease duration whereas H\&Y stages were compared with the Mann-Whitney-U Test.

Red flags with a specifity for MSA-P $>95 \%$ and a significantly higher frequency than in PD were selected for factor analysis to reduce the number of red flags required for diagnosis. Principal component analysis with Varimax rotation was applied to extract factors; the number of factors was chosen based on the scree plot. Sensitivity, specifity, positive (PPV), and negative (NPV) predictive value for MSA-P were calculated using presence of the resulting red flag categories in probable MSA-P and PD patients. Predictive diagnostic sensitivity of the EMSA Red Flags for "probable MSA-P" was then analyzed in the possible MSA-P group. 
TABLE 1. EMSA-SG red flag check list

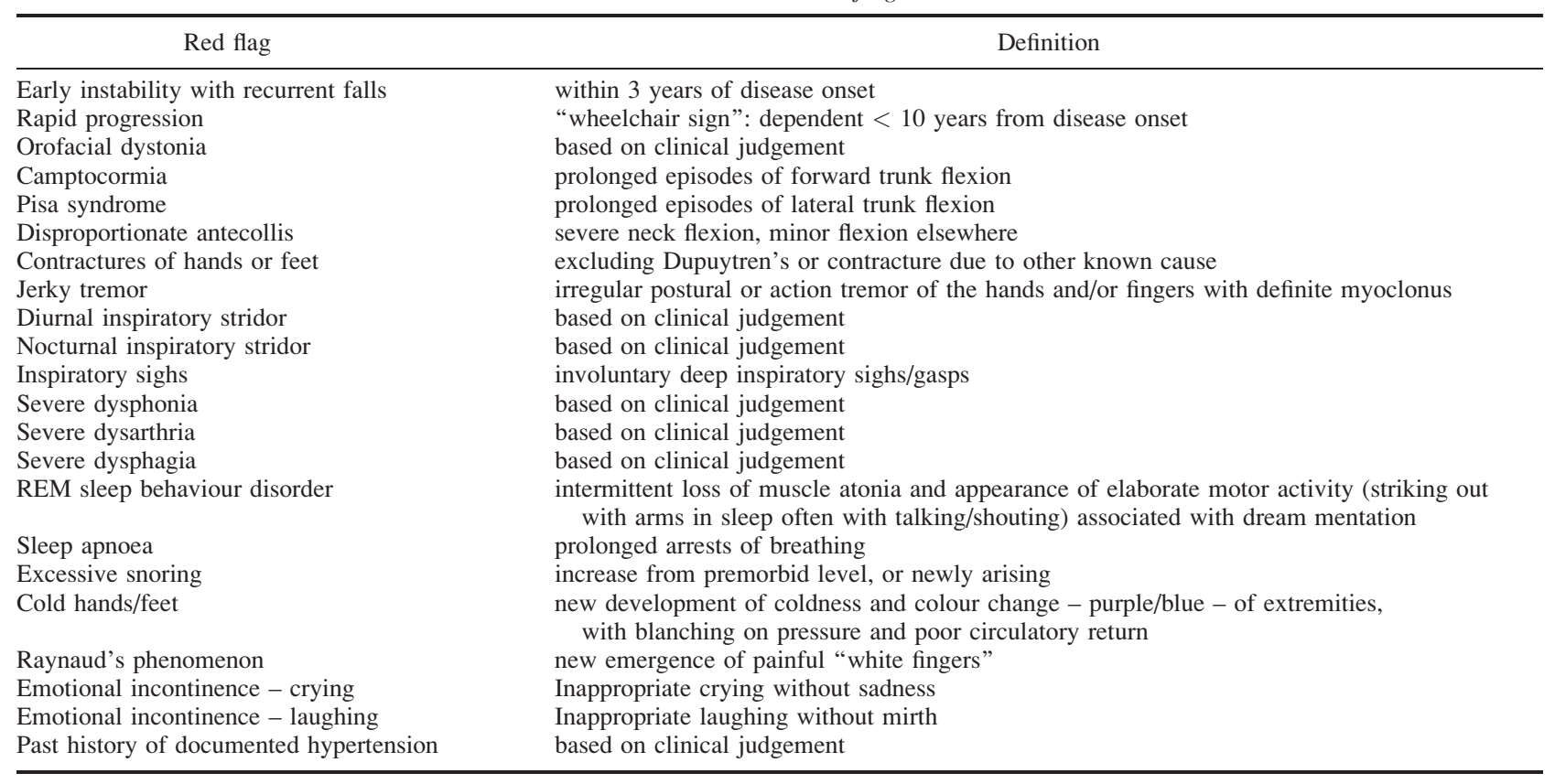

Red flags were recorded as follows: present, absent, unknown.

\section{RESULTS}

\section{Patient Characteristics}

Demographic and clinical data of MSA-P and PD patients are given in Table 2. There was no significant difference of age at examination $(P=0.46)$ and disease duration $(P=0.85)$ between groups. Hoehn and Yahr "off" stages were significantly higher in the MSA-P than in the PD group $(P<0.001)$.

\section{Red Flags}

The frequencies of potential red flags in MSA-P as compared with patients with PD are listed in Table 3. Those features with a specifity of $>95 \%$ and a significantly higher frequency than in PD patients were entered into the factor analysis.

Factor analysis was performed to reduce the number of red flags required for a diagnosis of probable MSA. Five factors emerged from this analysis explaining $73.3 \%$ of the variance. Early instability with recurrent falls within 3 years of onset loaded on the same factor as severe dysarthria, dysphonia, and dysphagia. Since these symptoms are not clinically related we decided to regard early instability as independent red flag category.

The resulting set of six red flag categories (Table 4) yielded $98.3 \%$ specificity and $84.2 \%$ sensitivity with a
PPV of $96 \%$ and a NPV of $92.7 \%$ if red flags from two or more categories were present.

By applying these criteria (at least one item in two out of six red flag categories) to the 17 patients with possible MSA-P who could be classified as having probable MSA-P on one of the follow-up visits, a diagnosis of probable MSA-P would have been possible in $13(76.5 \%)$ already at baseline, on average $15.9( \pm 7.0)$ months earlier than with the Consensus criteria alone.

\section{DISCUSSION}

Existing published diagnostic criteria ${ }^{1}$ for MSA perform better in terms of specificity than clinicians' diagnosis at first, but not last, visit. However, sensitivity is suboptimal. $^{7}$ Most clinicians therefore also consider the presence of additional warning signs, or red flags, when making a diagnosis of MSA in clinical practice. However, consensus criteria for these red flags are

TABLE 2. Patient characteristics

\begin{tabular}{lcccc}
\hline & MSA-P & PD & $P$-value & $\begin{array}{c}\text { Possible } \\
\text { MSA-P }\end{array}$ \\
\hline Age & $62.4 \pm 7.8$ & $64.2 \pm 8.2$ & n.s. & $63.3 \pm 10.5$ \\
Disease duration & $4.9 \pm 3.8$ & $5.2 \pm 3.7$ & n.s. & $3.9 \pm 1.8$ \\
m:f ratio & $1.4: 1$ & $1.5: 1$ & n.s. & $1.1: 1$ \\
Hoehn \& Yahr & $3.8(2.9-4.6)$ & $2.2(1.5-2.9)$ & $P<0.001$ & $3.7(2.9-4.5)$ \\
\hline
\end{tabular}


TABLE 3. Frequencies of red flags

\begin{tabular}{|c|c|c|c|c|}
\hline Red flag & MSA-P (\%) & $\mathrm{PD}(\%)$ & $P$-value & Specificity (\%) \\
\hline *Early instability & 67.9 & 2.6 & $<0.001$ & 97.4 \\
\hline *Rapid progression & 66.7 & 0.9 & $<0.001$ & 99.1 \\
\hline Camptocormia & 32.1 & 5.9 & $<0.001$ & 94.1 \\
\hline *Pisa syndrome & 42.1 & 2.5 & $<0.001$ & 97.5 \\
\hline *Disproportionate antecollis & 36.8 & 0.8 & $<0.001$ & 99.2 \\
\hline *Contractures of hands or feet & 15.8 & 3.4 & 0.010 & 96.6 \\
\hline Jerky tremor & 47.4 & 5.9 & $<0.001$ & 94.1 \\
\hline *Diurnal inspiratory stridor & 22.8 & 0.9 & $<0.001$ & 99.1 \\
\hline *Nocturnal inspiratory stridor & 37.7 & 2.6 & $<0.001$ & 97.4 \\
\hline *Inspiratory sighs & 43.6 & 3.4 & $<0.001$ & 96.6 \\
\hline *Severe dysphonia & 50.9 & 4.2 & $<0.001$ & 95.8 \\
\hline *Severe dysarthria & 49.1 & 4.3 & $<0.001$ & 95.7 \\
\hline *Severe dysphagia & 33.3 & 0.9 & $<0.001$ & 99.1 \\
\hline REM sleep behavior disorder & 43.1 & 27.4 & 0.047 & 72.6 \\
\hline Sleep apnoea & 19.2 & 5.7 & 0.012 & 94.3 \\
\hline Cold hands/feet & 39.3 & 12.8 & $<0.001$ & 87.2 \\
\hline Raynaud phenomenon & 11.1 & 3.4 & 0.075 & 96.6 \\
\hline *Emotional incontinence - crying without sadness & 26.3 & 2.6 & $<0.001$ & 97.4 \\
\hline *Emotional incontinence - laughing without mirth & 14.0 & 1.7 & 0.003 & 98.3 \\
\hline Past history of documented hypertension & 34.5 & 19.1 & 0.037 & 80.9 \\
\hline
\end{tabular}

*Red flags selected for further analysis.

lacking and their frequency and diagnostic validity in predicting MSA in its early stages are unknown. EMSA-SG has therefore operationalized the use of potential red flags for MSA (RFCL), excluding cardinal "core" diagnostic features (such as orthostatic hypotension, urinary incontinence/retention, L-dopa unresponsive parkinsonism, cerebellar ataxia, and pyramidal signs) which form part of the diagnostic criteria. ${ }^{8}$ We now report results of a cross-sectional multicenter study on the diagnostic value of red flags in a cohort of European MSA patients matched with patients with PD using the EMSA-SG RFCL.

All but one red flag (Raynaud's phenomenon) were significantly more common in MSA compared to patients with PD. Thirteen were highly specific for MSA ( $>95 \%$ specificity) suggesting potential diagnostic utility. These were grouped into six separate categories using factor analysis. The presence of red flags out of at least two of these six red flag categories gave a specificity of $98.3 \%$ and sensitivity of $82.4 \%$ compared to patients with PD. In addition to these results in established cases of probable MSA, these criteria also predicted the development of probable MSA-P in cases of possible MSA-P during further follow-up with a sensitivity of $76.5 \%$.

Early instability with recurrent falls during the first 3 years from disease onset was the most frequent feature in our cohort, present in $68 \%$ of the patients. In a clinical series of 100 patients with possible and proba- ble MSA postural instability was present in $93 \%$ of patients. ${ }^{9}$ Early instability with recurrent falls is also common in progressive supranuclear palsy (PSP). ${ }^{10-12}$ Among the different causes of parkinsonism, latencies to onset of falls are the shortest in PSP patients, intermediate in MSA, dementia with Lewy bodies (DLB), and corticobasal degeneration (CBD), and longest in PD. ${ }^{12}$ Taken alone, early instability does not allow a clinical diagnosis of MSA in a parkinsonian patient. However, its presence suggests an atypical parkinsonian disorder rather than PD.

Rapid progression was found in 2/3 of our MSA-P patients, whereas only $2.6 \%$ of PD patients showed this feature. Rapid progression was defined as wheelchair dependency within 10 years of disease onset. This latency may appear long at first sight, however,

TABLE 4. Red flag categories

Early instability
Rapid progression
Abnormal postures $^{\mathrm{a}}$
Bulbar dysfunction $^{\mathrm{b}}$
Respiratory dysfunction $^{\mathrm{c}}$
Emotional incontinence $^{\mathrm{d}}$

Category is positive if one or more symptoms present.

${ }^{a}$ Includes Pisa syndrome, disproportionate antecollis and/or contractures of hand or feet.

${ }^{\mathrm{b}}$ Includes severe dysphonia, dysarthria and/or dysphagia.

${ }^{\mathrm{c}}$ Includes diurnal or nocturnal inspiratory stridor and/or inspiratory sighs.

${ }^{\mathrm{d}}$ Includes inappropriate crying and/or laughing. 
symptoms suggestive of autonomic failure including urinary incontinence or orthostatic hypotension both of which often precede motor signs by many years were also allowed to define disease onset. Further, previous natural history studies suggest that most patients with PD do not reach a wheelchair-bound state within 10 years from disease onset. ${ }^{13}$ Finally, redefining rapid progression by reaching a wheelchair-bound state within 5 years from motor onset did not change the diagnostic validity of this feature (data not shown).

In a large Japanese study of patients with MSA median intervals from onset to aid-requiring walking, confinement to a wheelchair, bedridden state, and death were $3,5,8$, and 9 years, respectively. ${ }^{14}$ In that study, patients manifesting combined motor and autonomic involvement within 3 years of onset had a significantly increased risk of reaching advanced disease stages earlier and of poor survival. Rapid clinical deterioration (H\&Y V within 5 years of disease onset) or the "wheelchair sign" despite dopaminergic treatment, are recognized features of atypical parkinsonian disorders including MSA. H\&Y progression rates are significantly faster in atypical parkinsonian disorders (APDs) than in PD. ${ }^{11}$ UPDRS progression rates in 38 patients with MSA-P were tenfold increased compared to published rates in PD. ${ }^{15}$ In summary, rapid disease progression is a characteristic feature of MSA, but it fails to discriminate among the atypical parkinsonian disorders.

The red flag category "abnormal postures" includes Pisa syndrome, disproportionate antecollis and/or contractures of hand or feet (excluding Dupuytren's disease or contracture due to other known cause). Camptocormia was present in $6 \%$ of PD patients and excluded from factor analysis. Pisa syndrome was observed in $42 \%$ of our patients. This syndrome was originally described as a rare subacute dystonic reaction appearing 3-10 days after initiation of neuroleptic treatment that is characterized by an abnormal tonic lateral flexion of the trunk associated with some backward rotation; the head and neck may also be involved. ${ }^{16}$ A similar picture may also occur as a subtype of tardive dystonia. ${ }^{17}$ The term Pisa syndrome is also used to describe prolonged episodes of lateral flexion without exposure to neuroleptics. In MSA, progressive striatal degeneration may be the underlying pathologic substrate of this unusual dystonic picture. ${ }^{18}$ Pisa syndrome may also emerge in patients with otherwise classical PD, however, in contrast to other reports ${ }^{19}$ it was very rare in our PD cohort.

Antecollis has been described in MSA, DLB, and PD. ${ }^{20-22}$ While a disproportionate antecollis may appear rarely in $\mathrm{PD}$, it is relatively frequent in MSA. $^{4,23}$ A disproportionate antecollis was observed in $37 \%$ of our patients.

Whereas more frequent in $\mathrm{CBD}^{24}$ and MSA, ${ }^{4,23}$ contractures of hands or feet are not exclusively limited to APDs but may also complicate otherwise typical PD. ${ }^{25}$ In the present study, $15.8 \%$ of MSA-P patients and $3.4 \%$ of PD patients were affected.

Bulbar syndromes, including severe dysarthria, dysphonia, and/or dysphagia were found in one third to one half of MSA patients but in less than $5 \%$ of patients with PD. However, bulbar features are also prominent in other APDs including PSP, ${ }^{10} \mathrm{DLB}^{26}$ and less commonly CBD. ${ }^{27}$

Speech is more severely affected in MSA than in PD. ${ }^{2,28}$ Latency to onset of dysarthria was found to be significantly longer in PD than in the APDs, but does not help distinguish between different APDs. Median dysarthria latencies were reported to be short in PSP and MSA (24 months each), intermediate in CBD and DLB (40 and 42 months), and long in PD (84 months). ${ }^{3}$ Dysarthria as a presenting symptom has also been described in clinical series of $\mathrm{PSP}^{29}$ and CBD. ${ }^{30}$ Speech impairment in MSA patients is probably multifactorial, related to laryngeal dysfunction, akinesia/ rigidity of lips/tongue and palate, and respiratory dysfunction. As well as the low volume monotony of parkinsonism, a croaky, quivering, irregular, severely hypophonic or slurring dysarthria is "often so characteristic that the diagnosis can be suggested by listening to the patient on the telephone." 31 The dysarthria profile of MSA differs from that of PSP by the greater prominence of ataxic components and the lesser degrees of spasticity. ${ }^{32}$

In a previous series of 35 pathologically confirmed cases of patients with MSA dysphagia had been recorded in $51 \%$ of cases. ${ }^{33}$ In another study, ${ }^{3}$ latency to onset of dysphagia was significantly longer in PD than in APDs, but did not help distinguish between different APDs. Median dysphagia latencies were intermediate in PSP (42 months), DLB (43 months), CBD (64 months), and MSA (67 months), and long in PD (130 months). Dysphagia (or dysarthria) within 1 year of disease onset was a distinguishing feature for APDs (specificity, 100\%) but failed to further distinguish among the APDs.

Respiratory dysfunction presenting with deep involuntary sighs or inspiratory stridor, both diurnal and nocturnal were frequent in MSA and rare in patients with PD. However, other respiratory problems, such as excessive snoring or sleep apnoea, were not specific for MSA. Although the literature is scanty regarding 
deep involuntary sighs/gasps, it appears to be a common feature in probable MSA cases. Inspiratory stridor, especially at night, is commonly attributed to vocal cord paralysis, ${ }^{34}$ but might instead reflect dystonia of the vocal cords. ${ }^{35}$ Inspiratory stridor has been documented in $9-34 \%$ of MSA patients and could develop at any time point in the disease process. ${ }^{9,23,33}$ In contrast, stridor is very uncommon in PD, although it has rarely been described as a dyskinetic side effect of L-dopa treatment in apparent PD. ${ }^{36}$ The incidence of nocturnal stridor is significantly higher in MSA than in PD. ${ }^{37}$

Emotional incontinence was present in $30 \%$ of patients; pseudobulbar crying spells were more frequent $(28 \%)$ than inappropriate laughing (13\%). They are rarely seen in PD. ${ }^{2}$ However, pseudobulbar crying is even more common in PSP. ${ }^{38}$

To investigate sensitivity of red flag categories in patients with questionable MSA we applied these criteria in a group of 17 patients, who at the time of administration of the RFCL fulfilled criteria for possible MSA-P but not for probable MSA-P. All these patients were diagnosed as probable MSA-P at one of the follow-up visits.

Thirteen $(76.5 \%)$ of these 17 patients could have been correctly diagnosed much earlier (mean 16 months) as probable MSA-P if the present red flag categories had been applied. Red flag categories would enhance sensitivity of the Consensus criteria further without compromising specifity.

However, we acknowledge potential limitations. The study lacks a post-mortem confirmation. The clinical diagnosis of MSA served as gold-standard for assessing the validity of red flags. The definition of some red flags was based on clinical judgement alone without exact definition. Since we did not administer the RFCL to other APDs we could not determine the frequency and diagnostic validity of the potential red flags in distinguishing between MSA and these disorders and PD. Several of the selected red flag categories are recognized hallmark features of other APDs. Further comparative studies are therefore required to determine those red flags that may discriminate MSA from other APDs. Because of the limited numbers of possible MSA-P patients with follow-up data, both sensitivity and specifity of the red flag categories in early disease stages, including in patients not yet fulfilling criteria for possible MSA, have to be investigated prospectively in larger patient cohorts.

We conclude that there are distinct motor and non motor clinical features of MSA that are not formally part of already published clinical diagnostic criteria.
These warning signs or red flags are present with a varying frequencies ranging from 10 to almost $70 \%$. The present study suggests that a combination of two out of the six proposed red flag categories is highly specific with a good sensitivity when comparing MSA$\mathrm{P}$ and patients with PD. $76.5 \%$ of the patients diagnosed as possible MSA-P at baseline who fulfilled diagnostic criteria for probable MSA-P at one of the follow-up visits could have been diagnosed correctly already at baseline visit by applying these criteria. As a consequence, these red flag categories might be suitable to act as supportive criteria for the diagnosis of probable MSA.

\section{REFERENCES}

1. Gilman S, Low P, Quinn N, et al. Consensus statement on the diagnosis of multiple system atrophy. American Autonomic Society and American Academy of Neurology. Clin Auton Res 1998; 8:359-362.

2. Gouider-Khouja N, Vidailhet M, Bonnet AM, Pichon J, Agid Y. "Pure" striatonigral degeneration and Parkinson's disease: a comparative clinical study. Mov Disord 1995;10:288-294.

3. Muller J, Wenning GK, Verny M, et al. Progression of dysarthria and dysphagia in postmortem-confirmed parkinsonian disorders. Arch Neurol 2001;58:259-264.

4. Quinn N. Multiple system atrophy-the nature of the beast. J Neurol Neurosurg Psychiatry 1989;Suppl:78-89.

5. Quinn NP. How to diagnose multiple system atrophy. Mov Disord 2005;20 (Suppl 12):S5-S10.

6. Gibb WR, Lees AJ. The relevance of the Lewy body to the pathogenesis of idiopathic Parkinson's disease. J Neurol Neurosurg Psychiatry 1988;51:745-752.

7. Osaki Y, Wenning GK, Daniel SE, et al. Do published criteria improve clinical diagnostic accuracy in multiple system atrophy? Neurology 2002;59:1486-1491.

8. Geser F, Seppi K, Stampfer-Kountchev, M et al. The European Multiple System Atrophy-Study Group (EMSA-SG). J Neural Transm 2005; 112:1677-1686.

9. Wenning GK, Ben Shlomo Y, Magalhaes M, Daniel SE, Quinn NP. Clinical features and natural history of multiple system atrophy. An analysis of 100 cases. Brain 1994;117 (Part 4):835845 .

10. Litvan I, Mangone CA, McKee A, et al. Natural history of progressive supranuclear palsy (Steele-Richardson-Olszewski syndrome) and clinical predictors of survival: a clinicopathological study. J Neurol Neurosurg Psychiatry 1996;60:615-620.

11. Muller J, Wenning GK, Jellinger K, McKee A, Poewe W, Litvan I. Progression of Hoehn and Yahr stages in Parkinsonian disorders: a clinicopathologic study. Neurology 2000;55:888-891.

12. Wenning GK, Ebersbach G, Verny M, et al. Progression of falls in postmortem-confirmed parkinsonian disorders. Mov Disord 1999; 14:947-950.

13. Poewe WH, Wenning GK. The natural history of Parkinson's disease. Neurology 1996;47:S146-S152.

14. Watanabe H, Saito Y, Terao S, et al. Progression and prognosis in multiple system atrophy: an analysis of 230 Japanese patients. Brain 2002;125:1070-1083.

15. Seppi K, Yekhlef F, Diem A, et al. Progression of parkinsonism in multiple system atrophy. J Neurol 2005;252:91-96.

16. Reinertsen AM. [Pisa syndrome. Posture disorder in elderly patients as a side effect of treatment with neuroleptics]. Tidsskr Nor Laegeforen 1975;95:1212-1215. 
17. Suzuki T, Koizumi J, Moroji T, Sakuma K, Hori M, Hori T. Clinical characteristics of the Pisa syndrome. Acta Psychiatr Scand 1990;82:454-457.

18. Hozumi I, Piao YS, Inuzuka T, et al. Marked asymmetry of putaminal pathology in an MSA-P patient with Pisa syndrome. Mov Disord 2004; 19:470-472.

19. Yokochi F. Lateral flexion in Parkinson's disease and Pisa syndrome. J Neurol 2006;253 (Suppl 7):vii17-vii20.

20. Boesch SM, Wenning GK, Ransmayr G, Poewe W. Dystonia in multiple system atrophy. J Neurol Neurosurg Psychiatry 2002;72: 300-303.

21. Jorens PG, Eycken MP, Parizel GA, Martin JJ. Antecollis in parkinsonism. Lancet 1989;1:1320-1321.

22. Yoshiyama Y, Takama J, Hattori T. The dropped head sign in parkinsonism. J Neurol Sci 1999;167:22-25.

23. Wenning GK, Tison F, Ben Shlomo Y, Daniel SE, Quinn NP. Multiple system atrophy: a review of 203 pathologically proven cases. Mov Disord 1997;12:133-147.

24. Vanek Z, Jankovic J. Dystonia in corticobasal degeneration. Mov Disord 2001;16:252-257.

25. Hu MT, Bland J, Clough C, Ellis CM, Chaudhuri KR. Limb contractures in levodopa-responsive parkinsonism: a clinical and investigational study of seven new cases. J Neurol 1999;246:671-676.

26. Geser F, Wenning GK, Poewe W, McKeith I. How to diagnose dementia with Lewy bodies: state of the art. Mov Disord 2005;20 (Suppl 12):S11-S20.

27. Wenning GK, Litvan I, Jankovic J, et al. Natural history and survival of 14 patients with corticobasal degeneration confirmed at postmortem examination. J Neurol Neurosurg Psychiatry 1998;64: $184-189$.

28. Wenning GK, Ben Shlomo Y, Hughes A, Daniel SE, Lees A, Quinn NP. What clinical features are most useful to distinguish definite multiple system atrophy from Parkinson's disease? J Neurol Neurosurg Psychiatry 2000;68:434-440.

29. Tolosa E, Valldeoriola F, Marti MJ. Clinical diagnosis and diagnostic criteria of progressive supranuclear palsy (Steele-Richardson-Olszewski syndrome). J Neural Transm Suppl 1994;42:1531.

30. Rinne JO, Lee MS, Thompson PD, Marsden CD. Corticobasal degeneration. A clinical study of 36 cases. Brain 1994;117:11831196.

31. Caplan LR. Clinical features of sporadic (Dejerine-Thomas) olivopontocerebellar atrophy. Adv Neurol 1984;41:217-224.

32. Kluin KJ, Foster NL, Berent S, Gilman S. Perceptual analysis of speech disorders in progressive supranuclear palsy. Neurology 1993;43:563-566.

33. Wenning GK, Ben Shlomo Y, Magalhaes M, Daniel SE, Quinn NP. Clinicopathological study of 35 cases of multiple system atrophy. J Neurol Neurosurg Psychiatry 1995;58:160-166.

34. Hughes RG, Gibbin KP, Lowe J. Vocal fold abductor paralysis as a solitary and fatal manifestation of multiple system atrophy. J Laryngol Otol 1998;112:177-178.

35. Merlo IM, Occhini A, Pacchetti C, Alfonsi E. Not paralysis, but dystonia causes stridor in multiple system atrophy. Neurology 2002;58:649-652.

36. Corbin DO, Williams AC. Stridor during dystonic phases of Parkinson's disease. J Neurol Neurosurg Psychiatry 1987;50: 821-822.

37. Ghorayeb I, Yekhlef F, Chrysostome V, Balestre E, Bioulac B, Tison F. Sleep disorders and their determinants in multiple system atrophy. J Neurol Neurosurg Psychiatry 2002;72:798800 .

38. Menza MA, Cocchiola J, Golbe LI. Psychiatric symptoms in progressive supranuclear palsy. Psychosomatics 1995;36:550-554. 